\title{
Augmentation Procedures for the Maxillary Sinus: A Case Report with 10-year Follow-up
}

\author{
Jihad Abdallah \\ Teaching Staff and Implant Program Coordinator, Department of Oral and Maxillofacial Surgery \\ School of Dentistry, Beirut Arab University (BAU), Diplomate, American Board of Oral Implantology/Implant Dentistry (ABOI/ID), Fellow \\ American Academy of Implant Dentistry (AAID), Chicago, USA
}

Correspondence: Jihad Abdallah, Beirut Implant and Esthetic Dentistry Center (BIDC), Cairo Street Zakka Building, 2nd Floor Hamra, Beirut, Lebanon, Phone: +9613 334 004, e-mail: beirutidc@ hotmail.com

\begin{abstract}
A partially edentulous maxilla is treated with bilateral sinus lifts and implant placement. The maxilla presented with total pneumatization at the right and left molar area and partial pneumatization on the left premolar. Soft and hard tissue anatomy for lower arch was suitable for implant placement, while in the maxilla bone was not adequate for placing the implants. In the maxillary right and left molar areas, a lateral sinus lift procedure was needed before implant placement. While in the left premolar area, an internal sinus lift procedure could be performed immediately before implant placement. Panoramic radiographs were taken on regular basis to document the implant survival and crestal bone levels, which were maintained at 10-year follow-up.
\end{abstract}

Keywords: Maxillary sinus, Implants, Autogenous bone graft, Lateral sinus lift, Internal sinus lift.

\section{INTRODUCTION}

Sufficient bone quantity is required for dental implant placement. The maxillary posterior edentulous area presents a difficult situation in implantology when compared to other areas of the mouth. Bone quantity in the maxillary posterior edentulous area may be insufficient for dental implant placement because of the presence of the maxillary sinus. When teeth are extracted in the posterior maxilla, bone in that area is lost due to inferior expansion of the sinus involving the residual ridge area. ${ }^{2-9}$ This process is known as pneumatization of the maxillary sinus. Moreover, bone density in this area also decreases rapidly and is the least dense area of the maxilla. There are number of techniques and sinus lift procedures aimed at increasing bone quantity prior to implant placement. These techniques utilize bone graft material, the patient's own bone (autogenous bone), a range of commercially available materials (biomaterials) or a combination of the two. ${ }^{3}$ Tatum developed the technique in 1977, but Boyne and James were the first to publish an article and describe the technique in $1980 .{ }^{1}$ Implants can sometimes be placed simultaneous with the grafting procedure or it may be necessary to perform the grafting first with the implant placement being a second procedure performed several months later. Both processes and associated criteria will be described later. When contemplating sinus grafting, it is important to know the anatomy, be able to evaluate the maxillary sinus, understand the surgical management procedures and identify data related to long-term results. Short implants (5 to $8 \mathrm{~mm}$ ) may be as effective and cause fewer complications as longer implants placed using a more complex technique. ${ }^{4}$ Two different techniques of sinus augmentation are described in the literature: Direct and indirect. The indication for indirect sinus augmentation is a minimum bone thickness of $5 \mathrm{~mm}$ underneath the sinus; otherwise, the direct sinus floor augmentation or a 2-stage indirect augmentation technique must be implemented. However, Misch considered that 8 $\mathrm{mm}$ subantral bone height is the limit for the indirect sinus augmentation technique, 5 to $8 \mathrm{~mm}$ bone height is indicated for 1-stage direct augmentation with implants, and cases with less than $5 \mathrm{~mm}$ bone height are indicated for the 2-stage direct augmentation technique.

\section{CASE STUDY}

The patient needed several restorative treatments including fillings, root canal therapy, crowning, bilateral sinus lifts and implant placement. The mandible was in a Kennedy class I division, a bilateral free-end saddle with enough height for implant placement. The maxilla was a class I with a total pneumatization at the right and left molar area and partial pneumatization on the left premolar. Soft and hard tissue anatomy for lower arch was suitable for implant placement, while in the maxilla bone was not adequate for placing the implants. In the maxillary right and left molar areas, a lateral sinus lift procedure was needed before implant placement. While in the left premolar area, an internal sinus lift procedure could be performed immediately before implant placement.

The patient wanted to replace her missing teeth with fixed restorations. She could not withstand the discomfort of a partial denture or the fear that a partial denture would be one step away from a complete denture, which she 
considered to be too drastic for a 45-year-old. She also wanted to restore bilateral functionality in terms of ease of normal chewing on both sides. Porcelain-fused to metal crowns and bridges were selected as prosthetic restorations.

On 17 February, 1999, the patient presented for surgery on the upper left edentulous area. Following proper draping, local anesthesia was performed; sterile technique guidelines were followed. Once the patient was properly anesthetized, a palatal incision was performed connecting the distant aspect of maxillary tuberosity. This was followed by a vertical incision from the distal aspect of the canine to the canine eminence on the buccal plate of bone. The incisions were performed by using a no. 15 surgical blade. A 7 mm deep pilot hole was drilled using a $2 \mathrm{~mm}$ diameter pilot drill on the crest distal to the canine. Another $7 \mathrm{~mm}$ deep hole was created in the bone $6 \mathrm{~mm}$ away from the first one. A digital radiography was taken with the drills in place to confirm their proximity to the floor of the maxillary sinus (about $1 \mathrm{~mm}$ ). This working depth was sufficient for an internal sinus lift procedure. While performing the osteotomy, the pilot drill was set at 1000 rotations per minute and cooled by continuous irrigation with a $0.9 \%$ saline solution. This step was particularly important as it established the depth and angulation of the osteotomy.

After confirming the $1 \mathrm{~mm}$ proximity to the sinus by digital radiography, progressively increasing drill sizes were used at a depth of $7 \mathrm{~mm}$ (up to $4 \mathrm{~mm}$ diameter). Drills 2.5, 3 , 3.5, and 4 were set at $50 \mathrm{rpm}$, a speed too low to heat the bone, and as such required no irrigation. This allowed for collection of bone particles that could be used as autogenous bone graft material. Bone particles were collected in a sterile dappen dish, mixed with deproteinated bovine hydroxyapetite Bio-Oss ${ }^{\circledR}$ (Geistlich Pharma AG, Wolhusen, Switzerland), and used as bone graft material in both sinus lift procedures. After the osteotomies were performed, a curette was used to collect any remaining bone particles in both bony cavities. This prepped the sites for the greenstick fracture procedure.

A Bicon ${ }^{\mathrm{TM}}$ osteotome, $3.5 \mathrm{~mm}$ in diameter (less than the diameter of the osteotomy site preventing friction or side resistance during tapping), was introduced into the cavity till it touched the floor. A small surgical mallet was used to tap gently on the osteotome. The slight but sharp force used fractured the bony ceiling while keeping it attached to the sinus membrane. After removing the osteotome, the sockets were half-filled with bone graft mixture using a no. 0 amalgam carrier. Two $4.0 \times 11 \mathrm{~mm}$ HA-coated Bicon ${ }^{\mathrm{TM}}$ implants were then placed in the sockets and tapped in place using an assembled implant-seating tip on a straight handle and a small surgical mallet. Both implants were tapped intermittently so as to elevate the sinus floor on both sites simultaneously. As the implants were seated, they pushed the bone graft apically, which in turn elevated the sinus floor. Once the implant-seating tip reached the crest of the bone, each implant was properly seated.
After confirming the result of the procedure using digital radiography, the patient was ready for the second procedure, the lateral sinus lift.

Using a Brassler pear-shaped fine fluted bur (Bicon ${ }^{\mathrm{TM}}$ part \# 260-201-029), the lateral antral wall at the first molar position was osteomized 1 to $2 \mathrm{~mm}$ above the antral floor. The osteotomy was round in shape, and about 6 to $8 \mathrm{~mm}$ in diameter; it was carried through to the Schneiderian membrane without penetrating it. This was performed at a speed of $40,000 \mathrm{rpm}$ with $0.9 \%$ saline irrigation. Using a set of sinus lift curettes (no. 1 standard bend, no. 2 severe bend, no. 3 antraplasty elevator), the Schneiderian membrane was then carefully elevated from the antral floor inferiorly, anterior and posterior through the osteotomy site by keeping a dull curette in contact with the bone at all times during the elevation step. Using a rongeur, any available bone was harvested from the maxillary tuberosity, crushed and mixed with about 1 gm of Bio-Oss ${ }^{\circledR}$ and a small amount of saline. This mixture was packed against the medial wall of the sinus using a bone-carrying syringe. After cutting the two retracting sutures, the mucoperiosteal flap was repositioned. The incision was closed starting at the angle connecting the palatal and vertical flaps (i.e. at the canine palatally). The palatal incision was closed with a continuous running suture with a lock, making the closure watertight. The vertical incision was closed with four interrupted 4.0 Vicryl sutures.

After postoperative instructions were given, the patient was dismissed and asked to return in 10 days for check-up and suture removal.

On 20 October, 1999, the patient came in for second stage surgery related to the implants replacing the two premolars and for the placement of an implant in the grafted site, to replace the 1 st molar. While this procedure could have been performed 6 months after the first, the delay was on account of other treatments applied to the patient's mouth.

Following anesthesia, an incision was laid closer to the palatal aspect of the crest. The black polyethylene healing plugs covering each implant well were located and removed using a no. 110 reamer. Two guide pins placed in lieu of polyethylene plugs, allowed us to test for osseointegration by moving the guide pin laterally and confirming that the implants were not moving. This also allowed us to check for the emergence direction of each abutment to be used. Two zero-degree Bicon ${ }^{\mathrm{TM}}$ abutments were chosen since implants were placed in optimal vertical positions. A $4.0 \mathrm{~mm}$ sulcus reamer, corresponding to the diameter of the abutment to be used, was eased onto the guide pin in each submerged implant's locking taper well. The excess bone on top of each implants was removed to allow the abutments to be fully seated.

The chosen abutments ( $4 \mathrm{~mm}$ in diameter, $6.5 \mathrm{~mm}$ in height, $0^{\circ}$ in angulation) were placed and checked for proper 
positioning, including occlusal clearance, using a clear vacupress stent made on a stone duplicate of a waxed-up cast of the future finished case.

The abutments were modified extraorally using a Bicon $^{\mathrm{TM}}$ abutment prep holder and an abutment-cutting bur designed for this purpose. After verification of proper modification, the implant well and abutment post were flushed, cleaned and dried. The locking-taper post of the abutments was inserted into both implant wells. Both were permanently seated by impaction using the abutment-seating tip assembled on the straight handle and a surgical mallet. The placed abutments could now be used as guides for the direction of the osteotomy to be made for implant placement in the 1st left molar site.

A $12 \mathrm{~mm}$ deep pilot hole was drilled at a speed of 1000 rpm with $0.9 \%$ saline irrigation. After confirming depth adequacy via digital radiography, progressively increasing drill sizes were used (up to $4 \mathrm{~mm}$ diameter at the depth of $12 \mathrm{~mm}$ ). Drills 2.5, 3, 3.5 and 4 were used at $50 \mathrm{rpm}$ and without irrigation, which is not needed at this speed. Again, bone particles were collected in a sterile dappen dish and used as autogenous graft material to cover any potential bone defect after implant placement. This was especially useful around the cut polyethylene-healing plug of the implant. After the osteotomy was performed, a curette was used to collect any remaining bone particles in the bone cavity, preparing the site for implant placement. At this stage, the curette was also used to check for possible buccal perforation. A $4.0 \times 11.0 \mathrm{~mm}$ hydroxyapatite coated Bicon ${ }^{\mathrm{TM}}$ was placed in the intact buccal bone plate and fully seated using an implant seating-tip assembled on a straight handle and a surgical mallet. The plastic healing plug was then cut to bone level using a healing plug cutter. The healing plug was $2 \mathrm{~mm}$ in diameter, while the bone cavity was 4 $\mathrm{mm}$ in diameter. The bone graft collected during osteotomy was used to fill the bone cavity around the healing plug.

Before suturing the repositioned flap, two $4.0 \mathrm{~mm}$ emergence cuffs were placed on the two $4.0 \mathrm{~mm}$ abutments. After suturing the wound, the plastic emergence cuffs were incorporated into composite resin material using the vacupress stent to create temporary crowns on the two abutments. After removing any excess material and grinding the occlusal surface to ensure proper occlusion, the patient was given postoperative instructions and dismissed.

On 12 June, 2000, second stage surgery for the left maxillary molar was performed using exactly the same procedures described for second stage surgery for the premolars. The only difference was the use of a $5.0 \mathrm{~mm}$ diameter abutment (rather than $4.00 \mathrm{~mm}$ as in the premolars).

Upon dismissal, the patient was instructed to continue using chlorhexidine mouth rinse up to one week postsurgery. The $2 \mathrm{gm}$ of Amoxicillin and $600 \mathrm{mg}$ Ibuprofen tid prescribed before surgery were to be maintained for one week until the pain and swelling had stopped. The patient was asked to use ice packs in 10-minute intervals throughout the first 4 to 6 hours following surgery. She was also instructed to sleep with her head raised (2 pillows), refrain from blowing her nose, cough with her mouth open and avoid flu exposure. Swelling and discomfort are the most common postsurgical complications, in addition to infection, acute postoperative sinusitis. ${ }^{11}$ To decrease swelling, the patient was instructed to take warm saline water baths as often as she could from postoperative day 2 onwards. Highly nourished soft food was indicated for the first week following surgery.

During an 18 months period, and on different appointments, the patient had received extensive dental treatments, including root canal treatments for teeth 13,14 , 15, 31, 41 and 42; extraction with direct implant and bone grafting around teeth 43 and 44 ; a lateral sinus lift for the area of missing maxillary right molars; implants to replace 16, 34, 35, 36, 37, 43, 44, 45 and 46; abutments for all implants; temporary crown placement on all implant abutments; root canal treatment for teeth 15, 14 and 13.

The patient was called back into the clinic in August of the same year for final impressions. Temporary crowns were removed; soft tissues around the abutments replacing the two left maxillary premolars were left open due to the action of the emergence cuffs with no need for any retracting cord. A one-step technique was used for the impression. First, the heavy body was extruded from its cartridge, mounted in the gum, and the tray was filled. Immediately after that, the light body consistency, also from a cartridge, was syringed on the abutments. The tray was then placed on top and kept undisturbed for around 5 minutes before withdrawal from the patient's mouth.

An impression for the lower arch and a wax-bite registration were made according to regular crown and bridge procedures. The temporary crowns were reinserted pending the completion of the prosthesis. A week later, the metal copings were tried and their full seating was checked using a light body material as a fit-checker. After confirming proper seating Duralay ${ }^{\mathrm{TM}}$ acrylic resin was used for bite registration on metal copings intraorally. Pick-up impressions were made for upper and lower arches using imprint $\mathrm{II}^{\mathrm{TM}}$ as an impression material to enable accurate remounting by the laboratory technician. The ceramic bridges were tested a week later. Mesial proximal contacts were checked first to ensure proper seating and the occlusion was adjusted accordingly. Bridges were sent for glazing and temporarily cemented 2 days later. The patient was asked to return a week later for fine-tuning of the occlusion.

Following prosthetic restoration, the main concern is proper home care (provided that proper margins and embrasures with proper occlusion were respected in the fabrication of the prosthesis, which they were). The patient was thus instructed to control plaque accumulation using chlorhexidine mouthwash, anti-plaque toothpaste, interdental brushes and dental floss wherever applicable. The 
importance of proxa-brush was highlighted and the proper size was chosen for the patient. The patient was asked to visit the clinic regularly (every 4 months) for scaling and cleaning. A panoramic X-ray was taken on a yearly basis. When needed, periapicals were taken to verify bone level around implants.

\section{DISCUSSION}

The primary method of long-term evaluation of sinus grafts has been implant survival. Since various graft materials have
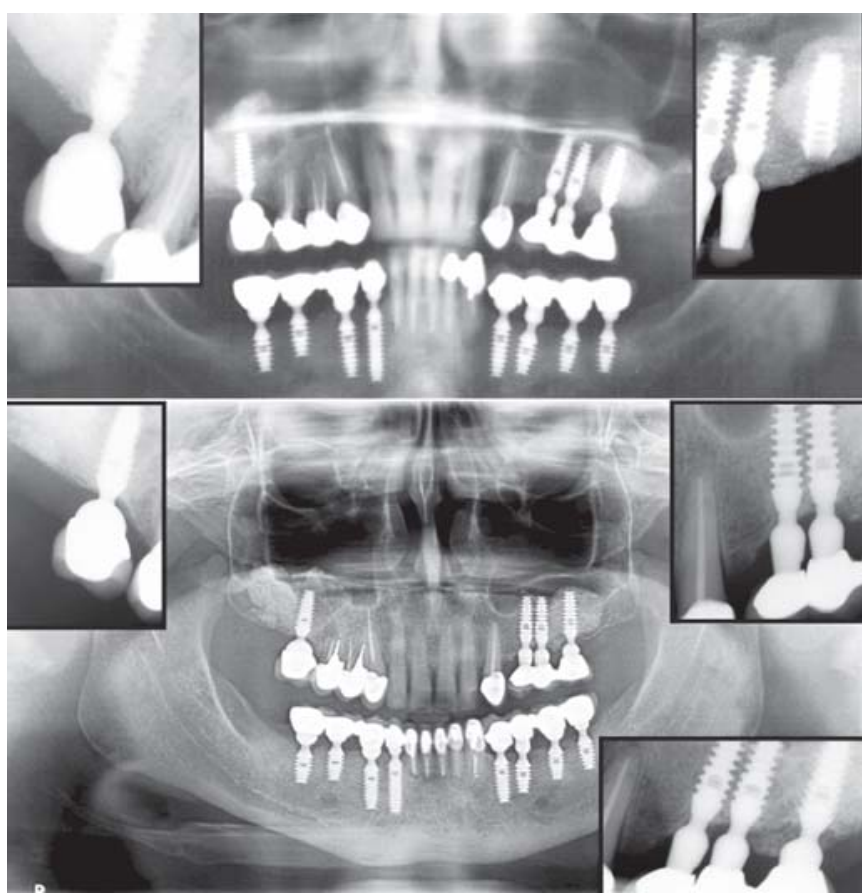

Fig. 1: Panoramic and periapical radiographs. Top radiographs were taken in 2000, and the lower radiographs were taken 10 years later in 2010

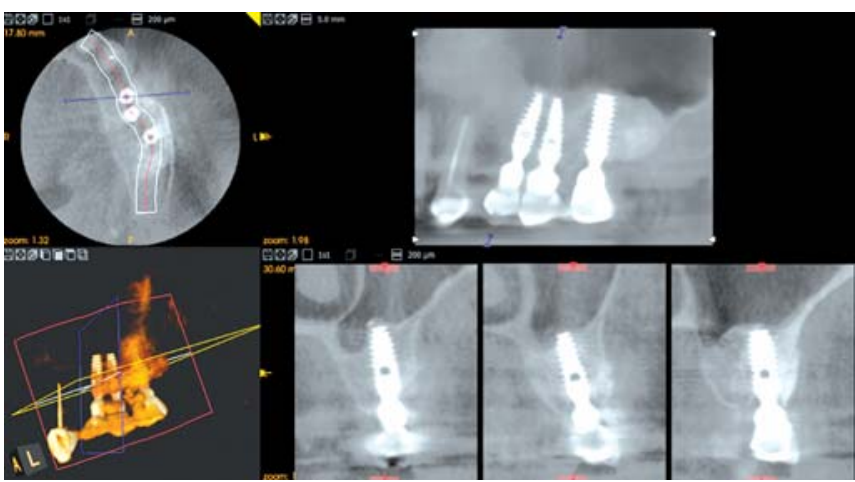

Fig. 2: CBCT taken 10 years later in 2010

been used in the sinus cavity along with dental implants of various shapes, surface coatings and placement protocols, the results reported varied among different authors. Overall, implants placed into the maxillary sinus area provide similar or slightly superior results than those placed in the same area without the sinus grafting procedure. Tidwell et al reported a high level of success when implants were placed into grafted sinuses. ${ }^{10}$ In our case, there was no crestal bone level changes either before loading or after loading for both lateral and internal sinus lift implants and the crestal bone levels stayed the same throughout the 10-year follow-up period (Figs 1 and 2). None of the implants showed any signs of mobility or peri-implant disease, and there were no exhibited sinus problems during the entire 10-year period (Figs 3A to D).

\section{CONCLUSION}

In this case implant survival in both internal and lateral sinus lift procedures is the same, in addition there was an observed
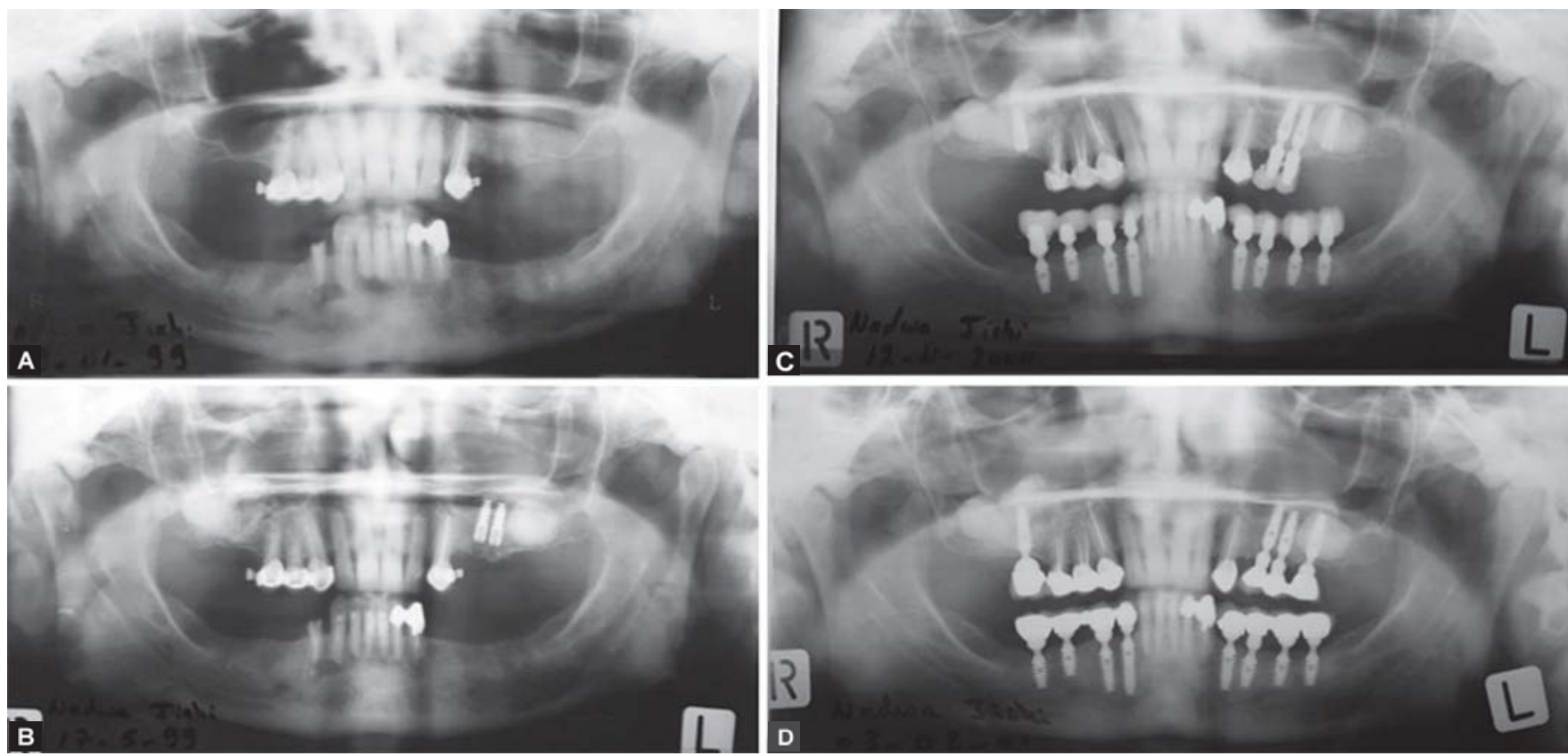

Figs 3A to D: (A) The preoperative panoramic radiograph, (B) the internal sinus graft elevation with simultaneous implant placement position no. 24 and 25 and both lateral sinus grafts with no implants placed, (C) the implant placement in position no. 16 and 26, (D) the postprosthetic radiograph 
stable crestal bone levels over a 10 -year period in the pressfit, fin-shaped, non-screw hydroxyapatite-coated implants.

\section{REFERENCES}

1. Boyne PJ, James RA. Grafting of the maxillary sinus floor with autologous marrow and bone. J Oral Surg 1980;38:613-16.

2. Esposito M, Grusovin MG, Rees J, Karasoulos D, Felice P, Alissa R, Worthington H, Coulthard P. Effectiveness of sinus lift procedures for dental implant rehabilitation: A Cochrane systematic review. Eur J Oral Implantol Spring 2010;3(1): 7-26.

3. Agamy EM, Niedermeier W. Indirect sinus floor elevation for osseointegrated prostheses: A 10-year prospective study. J Oral Implantol 2010;36(2):113-21.

4. Smiler DG, Johnson PW, Lozada JL, Misch C, Rosenlicht JL, Tatum OH, Wagner JR. Sinus lift grafts and endosseous implants: Treatment of the atrophic posterior maxilla. Dent Clin North Am 1992:36;151.
5. Kent JN, Block MS. Simultaneous maxillary sinus floor bone grafting and placement of hydroxyapatite-coated implants. J Oral Maxillofac Surg 1989;47:238-42.

6. Fugazzotto PA. Maxillary sinus grafting with and without simultaneous implant placement: Technical considerations and case reports. Int J Periodont Rest Dent 1994;14:544-51.

7. Summers RB. The osteotome techinque: Less invasive methods of elevating the sinus floor (Part 3). Compendium 1994;15:698, 700,702-704 passim;quiz 710.

8. Jensen OT, Shulman LB, Block MS, Iacono VJ. Report of the Sinus Consensus Conference of 1996. Int J Oral Maxillofac Implants 1998;13(Suppl):11-45.

9. Raja SV. Management of the posterior maxilla with sinus lift: Review of techniques. J Oral Maxillofac Surg Aug 2009; 67(8):1730-34.

10. Tidwell JK, Blijdorp PA, Stoelinga PJ, Briybs HB, Gubderks F. Composite grafting of the maxillary sinus for placement of endosteal implants: A preliminary report of 48 patients. Int J Oral Maxillofac Surg 1992;22:204-09.

11. Sandler NA, Johns FR, Braun TW. Advances in the management of acute and chronic sinusitis. J Oral Maxillofac Surg 1996;54:1005-13. 